\title{
Factores de riesgo de violencia escolar (bullying) severa en colegios privados de tres zonas de la sierra del Perú
}

\author{
Severe bullying risk factors in three Peruvian highland private schools
}

\author{
Isabel Amemiya', Miguel Oliveros², Armando Barrientos ${ }^{3}$ \\ ${ }^{1}$ Departamento Académico de Medicina Preventiva y Salud Pública, Facultad de Medicina, Universidad Nacional Mayor de San Marcos. Lima, Perú. \\ ${ }^{2}$ Departamento Académico de Pediatría, Facultad de Medicina, Universidad Nacional Mayor de San Marcos. Lima, Perú. \\ ${ }^{3}$ Ingeniero Estadístico. Unidad de Investigación, Instituto de Salud del Niño. Lima, Perú.
}

Resumen

Objetivo: Identificar los factores de riesgo de violencia escolar (bullying) severa en alumnos de colegios privados de tres zonas de la sierra del Perú. Diseño: Estudio tipo encuesta. Institución: Facultad de Medicina, Universidad Nacional Mayor de San Marcos, Lima, Perú. Participantes: Alumnos entre quinto de primaria y quinto de secundaria de colegios privados. Intervenciones: Una encuesta validada en estudios previos, para identificar violencia escolar (bullying), fue aplicada a 736 alumnos, entre quinto de primaria y quinto de secundaria, de colegios privados de Ayacucho, Huancavelica y Cusco (Sicuani). Se consideró bullying severo cuando contestaron positivamente 5 a más de las 9 opciones posibles de la pregunta que indagaba sobre los tipos de violencia. Se comparó 37 alumnos portadores de bullying severo con 74 alumnos tomados al azar del grupo que no tuvo violencia. Con análisis bivariado y multivariado de regresión logística se identificó factores asociados significativamente a la violencia escolar severa. Principales medidas de resultados: Factores de riesgo de violencia escolar (bullying) severa. Resultados: La violencia escolar severa se asoció significativamente con reacción de padres al conocer el hecho, repetición de la amenaza a pesar de comunicar las agresiones, presencia de pandilleros en el colegio, tener amigos pandilleros y poseer defecto físico. La regresión logística encontró asociación significativa con la reacción de los padres, presencia de pandilleros y poseer un defecto físico. Conclusiones: Se ha encontrado que la violencia escolar severa se asocia a múltiples factores de riesgo, que pueden y deben ser detectados precozmente, debido al daño psicológico que produce en los estudiantes.

Palabras clave: Violencia escolar; salud escolar; factores de riesgo.

\begin{abstract}
Objective: To identify severe bullying risk factors in three highland Peruvian zones private school students. Design: Survey type study. Setting: Faculty of Medicine, Universidad Nacional Mayor de San Marcos, Lima, Peru. Participants: Fifth elementary school to fifth high school private school students. Interventions: A survey validated in previous studies to identify school violence (bullying) was applied to 736 students from Ayacucho, Huancavelica and Cusco (Sicuani) private schools between fifth year of elementary school and fifth year of high school. We considered severe bullying when they answered positively five or more of the nine questions referring to types of bullying. We compared 37 students with severe bullying and 74 students randomly selected from the group not having bullying. With bivariate analyses and multivariate logistic regression analysis we identified factors significantly associated to severe bullying. Main outcome measures: Risk factors for severe bullying. Results: Severe bullying was significantly associated to parents' reaction when knowing the fact, repeated threatening in spite of bullying communication, presence of school gangs, having gang friends, and possessing a physical defect. Logistic regression found significant association with parents' reaction, gang presence, and possessing a physical defect. Conclusions: Severe bullying is associated to multiple risk factors that can and must be detected early because of the psychological damage generating in the students.
\end{abstract}

Key words: Bullying; school health; risk factors.

\section{INTRODUCCIÓN}

La violencia escolar (bullying) es un fenómeno antiguo en las escuelas, pero poco estudiado en nuestro medio. Se refiere al conjunto de comportamientos hostiles que una persona o grupo de personas, abusando de un poder real o ficticio, dirige contra un compañero/a de forma repetitiva y duradera, con la intención de causarle daño. En la mayoría de los casos, son comportamientos no encubiertos; el agresor no se esconde ni mantiene el anonimato y engloba agresiones directas e indirectas, tanto físicas como verbales, psicológicas y de exclusión social. La víctima está indefensa, no puede resolver por sí misma la situación y no existe una provocación previa por parte de la victima ${ }^{(1-3)}$.

La Organización Mundial de la Salud define la violencia como el uso deliberado de fuerza física o el poder, ya sea en grado de amenaza o efectivo, contra uno mismo, otra persona o un grupo o comunidad, que cause o tenga muchas probabilidades de causar lesiones, muerte, daños psicológicos, trastornos de muerte, del desarrollo o privaciones ${ }^{(1)}$.

Olweus (2), en 1983, definió: "Bullying es una conducta de persecución física y/o psicológica que realiza un alumno o alumna contra otro, al que escoge como víctima de repetidos ataques. Esta acción, negativa e intencionada, sitúa a la víctima en una posición de la que difícilmente puede escapar por sus propios medios. La continuidad de estas relaciones provoca en las víctimas efectos claramente negativos: ansiedad, descenso de la autoestima, y cuadros depresivos, que dificultan su integración en el medio escolar y el desarrollo normal de los aprendizajes."
Ortega ${ }^{(3)}$, en 1998, definió "El bullying es un comportamiento prolongado de insultos, rechazo social, intimidación y/o agresividad física de unos alumnos contra otros, que se convierten en víctimas de sus compañeros."

La escuela es una institución que es responsable de la formación y seguridad de sus estudiantes durante el tiempo que permanecen con ella. Los escolares están un promedio de 6 a 7 horas diarias, durante 5 días de la semana, en las escuelas, tiempo suficiente para crear un ámbito que puede repercutir positiva o negativamente en su vida futura. Muchas familias, debido a las exigencias laborales actuales, han dejado de cumplir, o cumplen muy precariamente, este rol formador y la han traspasado a las instituciones escolares, lo que asigna a los docentes funciones cada vez más complejas para formar a los 
estudiantes a enfrentar los desafíos de la modernidad y la globalización.

En nuestro país, se ha estudiado la violencia escolar en zonas de las tres regiones del Perú en las que existió violencia política, encontrado una incidencia de alrededor de 50\%. Se usó 9 ítems y se hizo el diagnóstico de bullying con la presencia de 2 o más de ellos. A continuación se clasificó esta violencia escolar en leve, moderada y severa, de acuerdo al número de ítems implicados ${ }^{(4,5)}$. La presencia de 5 o más de los 9 ítems positivos fue calificada como bullying severo, para identificar precozmente este problema, por las implicancias graves que de ella se pueden derivar, dígase violencia familiar, pérdida de autoestima, depresión y suicidio. Se detectó que la tercera parte de los padres no prestaba atención a las quejas de sus hijos, alcanzando en la región de la selva cifras cercanas al 50\%. Estas razones explicarían por qué $69 \%$ de los encuestados manifestó no comunicar el hecho a sus padres y preferir hacer la confidencia a los amigos ${ }^{(4,6)}$. Los maestros no son ajenos a la inacción y, en las regiones de la Costa y Sierra, las cifras están alrededor del $30 \%$, alcanzando en la región de la selva el $40 \%{ }^{(6)}$.

La violencia escolar puede ocasionar problemas en la salud física, emocional y social de la víctima. Alrededor de $20 \%$ de los encuestados necesitó asistencia médica ${ }^{(6)}$. Las víctimas de bullying a menudo comentan problemas para conciliar el sueño, enuresis, dolor abdominal, cefaleas y sentirse más tristes que sus compañeros ${ }^{(6,7)}$. Los alumnos que comunican victimización tienen 3 a 4 veces más probabilidades de presentar

Tabla 1. Ítems para el diagnóstico de violencia escolar (bullying) y escala de clasificación.

\begin{tabular}{ll}
\hline Ítems & \multicolumn{1}{c}{$\begin{array}{c}\text { Escala de clasificación de } \\
\text { diagnóstico de bullying }\end{array}$} \\
\hline $\begin{array}{l}\text { 1. Apodos } \\
\text { 2. Golpes }\end{array}$ & Bullying: Dos o más ítems positivos \\
3. Acoso & \\
\hline $\begin{array}{l}\text { 4. Insultos por correo-e } \\
\text { 5. Forzado a hacer cosas que no quiere }\end{array}$ & Leve: dos ítems o actos de acoso $(+)$ \\
6. Discriminado & Moderado: tres a cuatro ítems $(+)$ \\
$\begin{array}{l}\text { 7. Escupido } \\
\text { 8. Despojado }\end{array}$ & Severo: cinco o más ítems $(+)$ \\
9. Llamado homosexual & \\
\hline
\end{tabular}

síntomas de ansiedad que los niños no comprometidos ${ }^{(8,9)}$.

El bullying y el bullying severo deben ser considerados un problema de salud pública, por su magnitud y las consecuencias en la salud de la población, que merece ser estudiado a profundidad.

El objetivo de la investigación fue identificar factores de riesgo de violencia escolar (bullying) severa en alumnos de quinto de primaria a quinto de secundaria, de colegios privados de tres zonas de la Sierra del Perú, Ayacucho, Huancavelica y Cusco (Sicuani).

\section{MÉTODOS}

La UNMSM desarrolló un Programa de Atención Integral a Víctimas de la Violencia Política ${ }^{(5)}$. Esto ha permitido acercarnos a diversas comunidades y efectuar un estudio de la violencia escolar (bullying) en colegios nacionales y privados de Ayacucho, Cusco (Sicuani), Huancavelica, Junín (Satipo) y Lima Este, lugares en los que existió terrorismo. Los resultados de este artículo resumen el análisis de colegios privados de tres ciudades de la sierra del Perú.

Se realizó un estudio transversal, comparativo, en una muestra de 736 escolares de colegios privados de Ayacucho, Huancavelica y Cusco (Sicuani). Se usó un cuestionario validado en estudios previos ${ }^{(10)}$, que cuenta con 30 preguntas, de las cuales 29 son cerradas, que investigó al alumno como agresor, agredido u observador de los actos de intimidación; la conducta de los padres, maestros y autoridades, así como el manejo de este problema en el colegio y el entorno donde viven. Las preguntas fueron leídas y explicadas por un facilitador capacitado previamente. Se empleó además una guía construida para homogenizar la manera de leer y contestar las preguntas.

Se hizo el cálculo del tamaño de muestra mínimo para cada colegio, usando como parámetro de cálculo $60 \%$ de bullying, de acuerdo a un estudio previo ${ }^{(10)}$. Se escogió aleatoriamente las aulas, en las que se encuestó a todos los alumnos pertenecientes a ella, hasta cubrir la muestra calculada. Los alumnos habían sido sensibilizados e instruidos al respecto.

Para el diagnóstico de violencia escolar, se utilizó una pregunta con 9 opciones (ítems) de respuestas posibles, de las cuales 2 o más debían ser positivas (tabla 1). Se hizo una clasificación empírica de bullying en leve, moderado o severo, según el número de respuestas positivas: 2 respuestas positivas para el bullying leve, 3 a 4 para el moderado y 5 a más para el severo. Se detectó 37 alumnos con bullying severo (10,6\% de los casos de violencia escolar identificados). Se comparó el grupo de alumnos con bullying severo (grupo estudio) con un grupo de 74 alumnos tomado al azar, entre los alumnos que no tuvieron bullying (grupo comparativo).

Se determinó inicialmente los factores de riesgo con análisis bivariado y se calculó el OR y el intervalo de confianza (IC) de cada uno de ellos. Finalmente, un análisis multivariado de regresión logística identificó un modelo de explicación para la presencia de bullying severo.

Se protegió la confidencialidad, al ser las encuestas anónimas; se respetó la autonomía al dejar abierta la posibilidad de participar y se enfatizó la beneficencia al fortalecer valores como respeto, tolerancia y asertividad. Se solicitó autorización de las autoridades escolares explicando los beneficios que se podía obtener al conocer mejor el problema de violencia.

\section{RESULTADOS}

El 47,4\% de los alumnos encuestados manifestó violencia escolar; de estos, $10,6 \%$ (37 alumnos) presentó bullying severo. 
Tabla 2. Factores asociados significativamente a violencia severa (bullying) en escolares de colegios privados de Ayacucho, Huancavelica y Cusco (Sicuani).

\begin{tabular}{lccc}
\hline \multicolumn{1}{c}{ Factores } & $p$ & Odds ratio & IC al 95\% \\
\hline Reacción de padres & 0,005 & 4,13 & $1,61-10,6$ \\
Repetición de amenaza & 0,019 & 3,54 & $1,32-9,48$ \\
Presencia de pandilleros & 0,003 & 3,69 & $1,60-8,50$ \\
Amigos pandilleros & 0,026 & 3,49 & $1,26-9,65$ \\
Defecto físico & 0,010 & 3,23 & $1,39-7,50$ \\
Seguridad en el colegio & 0,026 & 0,37 & $0,16-0,84$ \\
\hline
\end{tabular}

Al comparar el grupo identificado como bullying severo (grupo estudio) con un grupo elegido al azar de 74 alumnos que no tuvo violencia escolar (grupo comparativo), en el análisis bivariado se encontró que la reacción de los padres al conocer el hecho, la repetición de la amenaza a pesar de haber comunicado las agresiones, la presencia de pandilleros en el recinto escolar, tener amigos pandilleros y poseer un defecto físico estuvieron significativamente asociados al bullying severo. La seguridad del colegio resultó factor protector (tabla 2).

En el análisis de regresión logística, el bullying severo se asoció significativamente con la reacción de los padres, presencia de pandilleros y poseer un defecto físico (tabla 3).

\section{DISCUSIÓN}

La ocurrencia de violencia escolar severa (bullying) se ha encontrado asociada a múltiples factores de riesgo.

El bullying severo ha sido poco mencionado, y se refiere más a la cronicidad de la presentación y complicaciones acompañantes, llámense depresión, pensamientos suicidas o suicidio ${ }^{(11-15)}$. Rodríguez menciona que el bullying puede ser incluido en las tipologías II (crónica) y III (mixta) para el trastorno de estrés postraumático, y se equipara a la violen-

Tabla 3. Regresión logística de factores asociados a violencia severa (bullying) en escolares de colegios privados de Ayacucho, Huancavelica y Cusco (Sicuani).

\begin{tabular}{lccc}
\hline \multicolumn{1}{c}{ Factores } & $p$ & Odds ratio & IC al 95\% \\
\hline Reacción de padres & 0,003 & 6,19 & $1,87-20,5$ \\
Presencia de pandilleros & 0,002 & 6,22 & $1,97-19,7$ \\
Defecto físico & 0,031 & 3,37 & $1,11-10,2$ \\
\hline
\end{tabular}

atención a las quejas de sus hijos, hecho que explica que $69 \%$ de los encuestados manifestó no comunicar el hecho a sus padres y preferir hacer la confidencia a los amigos ${ }^{(4,6)}$. Estos datos concuerdan con lo encontrado en este estudio, en el que la reacción de los padres fue un factor de riesgo de bullying severo. La escuela de padres, las reuniones de padres de familia, las actividades sociales entre las familias de los escolares pueden servir de instrumentos de intervención de este importante problema.

La existencia de pandillas en los colegios, así como el tener amigos pandilleros en el entorno hemos encontrado que son también factores de riesgo de violencia escolar severa. Los maestros deben ser ojos y oídos durante su labor diaria, para detectar e intervenir estos problemas latentes. Sin embargo, en un estudio nacional, los maestros no fueron ajenos a la inacción y en las regiones de la costa y sierra las cifras están alrededor de $30 \%{ }^{(6)}$. La ley del silencio y del dominio-sumisión se considera que son las que mantienen y perpetúan la violencia escolar.

En 1993, la Organización Panamericana de la Salud (OPS) y el Instituto Especializado de Rehabilitación (IER) determinaron que $13,08 \%$ de la población tenía una discapacidad. Según esta cifra, proyectada a la población estimada al 2002, existirían 969000 niños y adolescentes con discapacidad. El Ministerio de Educación estima que menos del 5\% asiste a la escuela ${ }^{(17)}$. Esta es la población expuesta al bullying severo, a consecuencia de su discapacidad, lo cual profundiza su ya vulnerable autoestima.

El alumno portador de algún defecto físico se encuentra más expuesto a la violencia escolar severa; en ellos se mezclan conductas de discriminación y exclusión para dañar la imagen social del niño y 'envenenar' a otros contra él. Se trata de presentar una imagen negativa, distorsionada y enfermiza de la víctima. Se critica todo cuanto hace o dice la víctima, y muchas veces contra lo que no ha dicho ni hecho. No importa lo que haga, todo es utilizado y sirve para inducir el rechazo de otros. El defecto físico en los alumnos fue mencionado en este estudio 3 veces más frecuentemente 
en el bullying severo que en el grupo sin bullying.

La violencia es hoy un componente cotidiano en nuestras vidas. Es una manifestación que ocurre en todos los niveles sociales, económicos y culturales. Esta se ha puesto de manifiesto también en las instituciones escolares. Dicha violencia fue ocultada, negada y silenciada durante muchos años por educadores y autoridades; pero, evitar y suprimir esos actos violentos no ha hecho más que empeorarlos. El problema debe ser tomado con firmeza y en toda su magnitud ${ }^{(4,6,10)}$.

Los resultados del presente trabajo no pueden ser extrapolados a otras poblaciones, debido a que la muestra fue seleccionada intencionalmente; sin embargo, considerando que se seleccionó los colegios más grandes e importantes de cada una de las ciudades estudiadas, se pudo observar que los docentes y alumnos de dichos colegios seleccionados estuvieron poco sensibilizados con el tema del bullying. A pesar de ello las encuestas fueron adecuadamente llenadas por los escolares y presentaron pocas respuestas en blanco. Finalmente, creemos que se requiere profundizar el conocimiento sobre el bullying. Para ello, recomendamos abordarlo desde una óptica cualitativa. También, debemos conocer la magnitud y características del bullying que se manifiesta a través de las tecnologías de información, el llamado cyberbullying.

En conclusión, la violencia escolar severa (bullying), es multicausal, puede y debe ser detectada, de manera de identificar temprana y precozmente los factores que ocasionan daño psicológico a los estudiantes. Esta es una acción que nos permitirá efectuar reparaciones en la salud mental, antes que continúe deteriorándose.

\section{AGRADECIMIENTOS}

A los Directores y docentes de los colegios participantes en la investigación y a los alumnos que colaboraron con el desarrollo de la encuesta.

\section{REFERENCIAS BIBLIOGRÁFICAS}

1. Acosoescolar.es. Definiciones de acoso escolar o bullying [Internet]. Madrid: Acosoescolar.es; 2008 [citado el 15 de agosto de 2009] Disponible en: http://www.acosoescolar.es/wp-content/ uploads/2008/01/definiciones.pdf

2. Olweus D. Bullying at school: what we know and what we can do. Oxford: Blackwell; 1993.

3. Ortega R. Relaciones interpersonales en la educación. El problema de la violencia escolar en el siglo que viene. Revista de Educación y Cultura. 1992;(14):23-6.

4. Oliveros M, Figueroa L, Mayorga G, Cano B, Quispe Y, Barrientos A. Violencia escolar (bullying) en Colegios Estatales de Primaria en el Perú. Rev Per Pediatr. 2008;61(4):215-20.

5. Facultad de Medicina, Universidad Nacional Mayor de San Marcos. Programa Permanente de Capacitación para la Atención Integral de las Víctimas de la Violencia 2006 - 2009. Lima: UNMSM; 2006.

6. Oliveros M, Figueroa L, Mayorga G, Cano G, Quispe Y, Barrientos A. Intimidación (bullying) en Colegios Estatales de Secundaria del Perú. Rev Per Pediat. (en prensa)

7. Piñuel I. Acoso y Violencia Escolar en España. Madrid: Ed. Heidi; 2007.

8. Pickett W, Craig W, Harel Y, Cunningham J, Simpson K, Molcho M, Mazur J, Dostaler S, Overpeck MD, Currie CE; HBSC Violence and Injuries Writing Group. Cross national study of fighting and weapon carrying as determinants of adolescent injury. Pediatrics. 2005;116(6):e855-63.
9. Riobó E. Redacción Aprendemas.com - Centro Reyna Sofía. Octubre de 2005.

10. Oliveros M, Barrientos A. Incidencia y factores de riesgo de la intimidación "bullying" en un colegio particular de Lima, Perú. Rev Per Pediatr. 2007;60(3):150-5.

11. Kim YS, Leventhal B. Bullying and suicide. A review. Int J Adolesc Med Health. 2008;20(2):133-54.

12. Kaltiala-HeinoR, Rimpela M, Marttunen M, Rimpela A, Rantanen P. Bullying, depression, and suicidal ideation in Finnish adolescents: School Survey. BMJ. 1999;319:348-51.

13. Salmon G, James A, Smith DM. Bullying in schools: self reported anxiety, depression, and self esteem in secondary school children. BMJ. 1998;317:924.

14. Glew GM, Fan MY, Katon W, Rivara FP, Kernic MA. Bullying, psychosocial adjustment, and academic performance in elementary school. Arch Pediatr Adolesc Med. 2005;159:1026-31.

15. Brunstein KA, Marrocco F, Kleinman M, Schonfeld IS, Gould MS. Bullying, depression, and suicidality in adolescents [Internet]. J Am Acad Child Adolesc Psychiatry. 2007;46(1):40-9.

16. Rodriguez Piedra R, Seoane Lago A, Pedreira Massa JL. [Children against children: bullying as an emerging disorder]. An Pediatr (Barc). 2006;64(2):162-6.

17. Congreso de la República del Perú. Comisión Especial de Estudio sobre Discapacidad del Congreso de la República (CEEDIS) [Internet]. Lima: Congreso de la República del Perú [citado el 17 agosto 2009]. Disponible en: http://www. congreso.gob.pe/comisiones/2002/discapacidad/ documentos/encarte-discapacidad-ojo.pdf

Manuscrito recibido el 19 de octubre de 2009 y aceptado para publicación el 23 de noviembre de 2009.

\section{Correspondencia}

Dra. Isabel Amemiya Hoshi

Huiracocha 1645

Lima 11, Perú

Correo-e: iamemiya2002@yahoo.com 